\title{
THE INFLUENCE OF MORPHOLOGICAL STRUCTURE ON THE PATHOLOGY OF JOINTS
}

\author{
BY R. G. ABERCROMBIE
}

NotwithSTANDING the great diversity of arthritic conditions, each joint in the body possesses a character of its own in its response to the invasion of morbid processes. This is partly due to the special mechanical factors of each particular joint, and partly to the morphological structure which is the heritage of its evolutionary history.

Human anatomy may be regarded as the contemporary phase of a long series of modifications and adaptations of function and of form which have left their traces upon every organ and structure of the body. With regard to the skeleton and the motor structures generally, the all-important transition is that which has led to the assumption of the erect posture, for this transition is comparatively recent in time and has involved far-reaching changes in the architecture and the mechanism of the bones, joints, muscles, and ligaments.

The attempt to unravel the ancestry of the human race may be compared to the attempt to trace the genealogy of an individual man. The first few generations in the lineage of an individual may be traced backwards with confidence. As we follow the line of descent still further into the past, it becomes progressively more obscure. Nevertheless, from a variety of considerations we may still obtain a general idea as to the remote origin and the stock from which the family arose. Similarly, with regard to the evolution of the human species there is a general agreement as to the more recent stages, but the more remotely the line is traced, the more debatable does it become.

Our knowledge of man's ancestry is largely derived from the geological record. Light is also thrown on the problem by the study of embryology and development, for the "Law of Recapitulation " states that each individual during its development passes through the successive phases which the race to which it belongs has passed through during its evolution. A further 
sidelight is obtained from the study of existing forms of life which are presumed to bear a resemblance to ancestral types now extinct.

As the result of all these lines of investigation, it is generally believed that the human species is descended from a prehuman type which had much of the character of the anthropoid apes of today. Beyond that point the lineage becomes obscure. It is surmised that earlier progenitors were of an ape-like form whose habitual posture approximated to the quadrupedal, and that a still more remote ancestor was of a prosimian type comparable to the present-day tarsier. Although it may well be believed that the convergence of the eyes in the prosimian ancestry resembled that seen in the tarsier, it is probable that the resemblance of bodily structure was only of a general nature, for the existing tarsier is a highly specialised animal with strangely elongated tarsal bones. Since the dentition of the tarsier is insectivorous, it has been conjectured that man's remote prosimian ancestor was derived from the insectivores, perhaps from some type resembling the tupaia or tree-shrew, and earlier still from a marsupial. Man's lineage is thus conjecturally traced backwards through the anthropoid type to the prosimian and beyond that to the quadruped. Imperfect though our knowledge is, it is yet sufficient to add interest to the study of the pathology of the joints, for the sequence of evolutionary development has an influence on the sequence of pathological events.

It is a generally accepted principle that it is the most recently acquired characters that are the most precarious in their tenure when exposed to the influence of disease. A similar principle is expressed by the "Law of Dissolution" in pharmacology, which states that the toxic effects of such substances as alcohol and chloroform first impair those functions which have been the last to appear in the history of the species, and that as the toxic effects progressively increase, less recently acquired functions are affected in order of succession, the most primitive functions being affected last of all. Comparable effects may sometimes be observed as the result of the gradual encroachments of a progressive morbid process upon the structure and function of joints. The advance of a chronic disease may thus reproduce, in a reverse order, the successive phases which the affected structures have passed through during the course of their evolution. To this process the term retrogression may be applied. 
In order to apply these ideas to the pathology of a particular joint-the shoulder-joint, for example-it is necessary to consider the characters of the joint in question as exemplified in a series of existing animals, each one of which typifies a stage in human evolution. Such a series is constituted by the quadruped, the prosimian, the anthropoid and man.

In the quadrupedal type in general the head of the humerus is relatively small, and is proportionate to the size of the glenoid cavity. It is connected with the shaft by a well-defined anatomical neck. The articular surface is restricted in size, oval in form and antero-posterior in direction, and is thus specially adapted to the movements of flexion and extension. Abduction is poorly provided for by the shape of the joint surfaces and is imperfectly accomplished by movements of the scapula on the chest wall.

In the prosimians the head of the humerus is larger in relation to the size of the glenoid cavity, the anatomical neck is less defined, and the articular surface of the head is more extensive. More abduction is possible than in the quadruped, and in some species it is performed freely; but from the form of the skeleton it appears to be chiefly carried out by movements of the scapula upon the thorax rather than by movement at the shoulder-joint. Little information is to be obtained from the shoulder-joint of the tarsier, for it is imperfectly developed and specialised for certain peculiar movements. In the lower monkeys the general characters of the joint still have a quadrupedal character; abduction appears to be performed partly at the joint itself and partly by scapular movement, reinforced by nimble sideways movements of the whole trunk.

In the anthropoid apes the transformation to the human type of shoulder is complete. The head of the humerus is disproportionately large in relation to the glenoid cavity; it is hemispherical in form and the anatomical neck is obscured. The extensive articular surface allows for an extremely wide range of movement, including abduction. In the gibbon, abduction appears to be more developed than in man, so that the animal moves its upper limbs in every direction with the utmost freedom, without adjustment of the position of its trunk. A clue to the ancestry is provided by the observation that in the skeleton of the immature chimpanzee the head of the humerus is of distinctly quadrupedal type, and if a juvenile chimpanzee is watched at play the deficiency of abduction is clearly apparent. 
In the human embryo the head of the humerus is also of quadrupedal form.*

The structure of the shoulder-joint in man resembles that of the anthropoid apes, but there is a distinction with regard to function. In the ape the grasping and "handling" of objects is distributed amongst all four limbs; in man the lower limbs are degraded to the position of pediments, and all exact movements are performed by the upper. The special functions of man's shoulder are thus not only recently acquired, but are subjected to the strain of continuous usage.

The characters of the shoulder-joint, as studied in the quadruped, the prosimian, the anthropoid, and finally in man, thus represent a series of modifications whereby an articulation adapted to carry the weight of the body has been gradually transformed into a highly mobile joint well fitted to execute varied and accurate movements, including abduction at the shoulder-joint itself. Abduction at the shoulder-joint is of importance in that it confers upon the movements of the upper limb an accuracy far greater than can be attained by similar movements carried out by the rotation of the scapula or by adjustment of the position of the trunk. The advance in articular abduction is thus an advance in accuracy of movement.

In man, both types of abduction come into play. The movement of the arm from the position of adduction through ninety degrees to that of horizontal abduction is carried out at the shoulder-joint, whereas the movement through the ensuing ninety degrees to the vertical is effected by rotation of the scapula. Extreme extension also entails a change in the position of the scapula, though this is small in degree compared to that associated with extreme abduction. Accurate movements of the upper extremity in man are best performed with the limb in a position below that of the horizontal, where they are controlled by the shoulderjoint; whereas movements performed with the limb in the position above the horizontal are controlled by the more primitive rotation of the scapula and are in consequence clumsy in character.

The shoulder-joint affords a good illustration of the influence of the evolutionary sequence upon pathology, and it is therefore

* The above observations are largely based on the study of the excellent osteological series illustrating the ancestry of man in the Western Park Museum, Sheffield. 
of interest to consider in detail the morbid conditions to which it is subject. Since abduction at the joint itself is a recently acquired function, the muscles which subserve this movement are particularly liable to suffer from the effects of injury or disease. Paralysis of the deltoid often occurs as the result of an injury to the circumflex nerve or a toxic neuritis; and both the deltoid and the supraspinatus are frequently affected in acute anterior poliomyelitis, in birth paralysis and in traction paralysis of the brachial plexus. The tendon of the supraspinatus may become the seat of a degenerative or inflammatory process, particularly where it is related to the subacromial bursa, and the same tendon occasionally suffers rupture. The deltoid is often the subject of muscular rheumatism, and its insertion into the humerus is a frequent site of tenderness, due either to a rheumatic or fibrositic process or as the result of strain.

The periarticular fibrous and connective tissues about the shoulder have undergone structural modifications which increase their pliability and mobility, and thus facilitate the newly acquired and complex muscular movements. These recent changes in the connective tissues are insecure in their tenure. As the result of a large number of diverse pathological influences they tend to retrogression, and thus give rise to a painful and progressive stiffness of the shoulder-joint. Several names have been suggested for this condition, such as "scapulo-humeral periarthritis" (Douthwaite); "frozen shoulder" (Codman); "Duplay's disease," from the name of the author who first accurately described the condition. "Periarticular fibrositis of the shoulder" is here suggested as an appropriate designation, the term fibrositis being included for reasons which will appear later.

The various pathological conditions which may give rise to the condition are as follows:

1. Trauma; direct injuries or strains of the joint itself or of its neighbourhood.

2. Rheumatism, using the term in its most general sense, including general arthritic conditions and menopausal rheumatism.

3. Infective conditions, such as pneumonia, influenza, tonsillitis and cholecystitis. Latent infections also sometimes appear to operate as a cause.

4. Neuropathic conditions. In hemiplegia, obscure trophic 
influences associated with the disuse may give rise to painful stiffness of the shoulder.

5. Debility, chronic ill-health, cachexia, severe anæmia, metabolic affections, such as glycosuria or gout.

6. Immobility or inaction from any cause. For example, when plaster-of-Paris splinting has been used for fracture of any part of the upper extremity, the shoulder may remain troublesome for long after the recovery of the fracture.

The pathological causation is thus very diverse, but with regard to the affection of the shoulder-joint the cases resemble each other closely in their symptoms, physical signs and clinical course; it is therefore a fair assumption that though the pathology is diverse, the morbid anatomy is a constant one. Our knowledge of the morbid anatomy is largely based on an autopsy described by Duplay and quoted by Douthwaite (B.M.J., February 26, 1938).

The case had been treated by Duplay for a periarthritis of the shoulder following an injury. The result was good, but the patient died two months later of pneumonia. The results of the autopsy thus disclosed the vestiges of the morbid condition rather than its active phase. "On the affected side the deltoid was paler than normal; the subdeltoid tissue was fibrous, exhibiting dense bands running to the upper end of the humerus. The subacromial bursa was obliterated. The tendons of the supraand infraspinati had lost their polish, as had also the inferior surface of the acromion. It was evident that the subacromial bursitis was the chief lesion, with extension to neighbouring structures. There could be seen ruptured adhesions on the inferior surface of the deltoid. The articular capsule was somewhat thickened, but the articular surfaces were quite normal." It is clear that the morbid process had been essentially a periarticular fibrositis affecting the connective tissues surrounding the joint, but not the articulation itself. This accords with the clinical evidence, for the skiagraphic appearances of the bones are normal, there is no crepitus on movement, and muscular wasting is slight or absent. Further evidence of a widespread fibrositic process is afforded during the acute phase by the presence of points of tenderness, the most usual sites being the insertion of the deltoid, the tip of the coracoid process and the supra- and infraspinous fossæ. The subacromial bursa, a structure subserving the recently acquired abduction, is frequently 
but not invariably affected. Since no swelling occurs, either of the bursa or elsewhere, the process appears to partake as much of a degenerative as of an inflammatory character.

The existence of a dual or alternative mechanism for the movements of the shoulder, particularly abduction, explains the insidious manner in which the impairment of movement often occurs. The first slight loss of articular movement is easily compensated for by the more primitive mechanism of scapula movements, and thus may pass unnoticed; once initiated, the process tends to progress, greater proportions of the movements being gradually transferred to the scapula without the patient's knowledge. Finally, a degree of stiffness is reached which cannot be compensated for by scapular movements, and the patient finds to his surprise that he is unable to perform some such action as putting his hand in his pocket. (In such a joint as the elbow, for the sake of comparison, flexion and extension must be performed at the joint itself or not at all, and thus the natural movements tend to check the progress of an insidious ankylosing process.)

Each successive phase in the loss of movement represents a stage in retrogression. During the first phase, abduction at the shoulder-joint is impaired and is transferred to movements of the scapula, so that at this stage the patient is in the condition of a prosimian. As the process advances, abduction at the shoulder-joint is abolished altogether, and the loss can no longer be compensated by the scapular movements; although abduction is lost, the patient can still perform flexion and extension, so that at this stage he is in the condition of a quadruped.

These observations have a bearing on the treatment. If a patient whose arm is in the quadrupedal condition attempts to move the limb while he retains the ordinary human posture, sitting or standing, his efforts are both difficult and painful; whereas if he adopts the posture of a quadruped, the movements are easy and painless. To utilise this in practice, the patient should support himself by placing his sound forearm and hand on the edge of a table, and should flex the trunk forwards at the hips, the affected limb being allowed to dangle vertically downwards. In this quadrupedal position he should swing his dangling arm, gently at first, and more freely as the range of movement improves. Flexion and extension are first performed, and abduction, adduction and circumduction are gradually added, 
all the movements being aided by the pendulum-like swing of the pendent arm. Since the patient himself regulates the range of the movements, they are painless. With improvement in abduction, the later stages of treatment are carried out in the ordinary sitting or standing posture.

Another method of treatment may be alluded to, since it also depends on pathological considerations. The loss of pliability in the connective tissues impairs the normal laxity of the joint and thus impedes its natural play. Improvement may often be obtained by the simple process of stretching the affected tissues. The patient's position being fixed by some simple device, the manipulator grasps the hand of the affected limb and brings the arm into the position of abduction. Steady traction is then made upon the hand, thus bringing all the structures about the shoulder into a state of tension. No movements should be used, and care should be taken to relax the tension gradually, or pain will ensue. It is not necessary to discuss here the value of the various methods of physical treatment, or of forced movements under anæsthesia; all such methods have their place in the treatment of suitably selected cases. The essential object of treatment is to restore the movements of the joint, for the restoration of movement is associated with a regeneration of the normal structure and function of the connective tissues. Immobilisation may be advisable for a period during the acute phase, but it should not be too prolonged.

The shoulder-joint is occasionally the subject of osteo-arthritis. This condition is far less frequent than periarticular fibrositis, and clinically the two conditions form a complete contrast. In the osteo-arthritic condition the limitation of movement is usually of only moderate grade, creaking and crackling are present on movement, muscular wasting is well marked, the $\mathrm{X}$-ray examination frequently discloses an irregularity of the bones, sometimes associated with the presence of minute osteophytes, the pain is often characteristically nocturnal, and the treatment required is of a lenitive character. Rarely periarticular fibrositis may coexist with an osteo-arthritic condition. Osteo-arthritis of the shoulder is most usually seen in elderly subjects, particularly those engaged in laborious occupations, and it not infrequently follows trauma. It may be regarded as a degenerative process akin to the similar affection of the hipjoint. 
The evolutionary history of the hip-joint differs widely from that of the shoulder, and it is therefore in accordance with expectation that the pathological reactions of the two structures should be dissimilar. The hip-joint in man retains its primitive functions of transmitting the weight of the body to the lower limbs and of subserving locomotion. These functions, however, have undergone modification in one respect: in the quadruped the functions in question are distributed amongst all four limbs, whereas in the prosimian, and to a still further extent in the anthropoid, they are largely relegated to the lower limbs. In man this specialisation is carried a stage further, so that the whole onus of weight-carrying and of locomotion falls upon the lower limbs, and in consequence upon the hip-joints. Hypertrophic changes, osteophytes and osteo-arthritis provide the evidence of the instability of a recent adaptation, and may be regarded as Nature's efforts to buttress up structures which are unequal to the strains to which they are subjected. Osteoarthritis, tuberculosis, osteo-chondritis juvenilis (Perthes' disease), slipping of the epiphysis and coxa vara are all associated either directly or indirectly with strain or trauma, and all are usual affections of the hip-joint, whereas similar or comparable conditions are much more rare in relation to the shoulder. In the hip the recent modification has been in the direction of increased stress, and the characteristic morbid changes are to be found in the bones and the joint itself; whereas in the shoulder the recent modification has been in the direction of increased mobility, and the characteristic morbid changes affect the connective tissues subserving this advance.

The transition in bodily posture from the horizontal to the vertical has been brought about by a modification of the architectural curvature of the vertebral column, and the chief element in this change has been the development of a curve with the convexity forwards in the lumbar region. In the prosimian the thoracic and lumbar vertebræ form a single large arch with the convexity backwards, and in the lower monkeys the curvature is of similar type but of less degree. The first indication of a lumbar curve convex forwards is to be seen in the anthropoid apes; it is faintly present in the skeleton of the orang-utang, distinctly visible in the gorilla, and in the chimpanzee there is an approximation to the human character of spine, with a sigmoid curve convex backwards in the thoracic and convex forwards 
in the lumbar region. In man the lumbar spine is strongly arched with the convexity forwards, so that the backward inclination of the upper limb of the arch carries the trunk backwards into the fully erect posture.

The successive modifications of posture have been associated with successive redistributions of stress. In the quadruped and the prosimian the weight of the body is distributed over the whole length of the spine; in the anthropoid it falls in a large proportion on the lumbar region, and in man the whole weight of the head, the upper limbs and the trunk is ultimately transmitted through the lower lumbar vertebræ.

Man's lumbar spine is thus subjected to the twofold modification of increased curvature and additional stress. The great variability of its structure indicates that it is still undergoing adaptive differentiation, and that its contemporary phase is one of plastic evolution. No other region of the body exhibits so gross a range of variation. The vertebræ which compose it may vary in number; the fifth may have a partially sacral character; the bodies or the laminæ may be imperfectly developed; the lateral processes may be detached; and the individual vertebræ, particularly the fifth, vary in magnitude and shape.

The variability of structure is associated with instability of function, which is expressed in the form of the symptoms of disease. Pain in the lower part of the back is one of the most frequent of human complaints. The symptoms may have many origins and may take many forms, physical and psychological. The lumbar spine is the most frequent site in the body for osteoarthritis; the adjacent muscles are the subjects of myalgia, and the connective tissues of fibrositis; lumbar and sacro-iliac subluxations and strains are often the legacies of slight developmental defects; and in debility and abnormal psychological states the maintenance of the curvature may rise into consciousness in the form of pain.

The shoulder, the hip and the spine have been taken as examples to illustrate the influence of evolutionary history and morphology upon pathology; and in many other regions of the body similar considerations serve to elucidate phenomena which would be otherwise anomalous and obscure. 